\title{
Uso de plantas medicinales y fitoterápicos en pacientes con Diabetes Mellitus tipo 2
}

\author{
*Patricia Acosta-Recalde, Gladys Lugo, Zully Vera, Macarena Morinigo, Gladys Mabel Maidana, \\ Lourdes Samaniego \\ Universidad Nacional de Asunción, Facultad de Ciencias Químicas, Departamento de Farmacia. Paraguay
}

Cómo referenciar este artículo/

How to reference this article:
Acosta-Recalde P, Lugo G, Vera Z, Morinigo M, Maidana GM, Samaniego L. Uso de plantas medicinales y fitoterápicos en pacientes con Diabetes Mellitus tipo 2. Mem. Inst. Investig. Cienc. Salud. 2018; 16(2): 6-11

\section{R E S U M E N}

Un gran número de especies vegetales son utilizados con fines medicinales para tratar diferentes patologías y entre ellas la diabetes que en el 2015 presentaba la mayor carga económica a los países de América Latina y el Caribe. A su vez, para la OMS, el uso de las plantas medicinales constituye una terapia más natural, más inocua, efectiva, de un costo racional y asequible a las poblaciones, por ende, para establecer su uso seguro es necesaria su correcta identificación. El objetivo del estudio fue describir las plantas medicinales y fitoterápicos empleadas por pacientes con diabetes mellitus tipo 2, para lo cual se realizó un estudio descriptivo de corte transverso, que incluyó a 41 pacientes registrados en el Programa Nacional de Diabetes por consulta regular en enero del 2015. Más de la mitad de la población manifestó consumir algún tipo de planta medicinal para el tratamiento de la diabetes mellitus tipo 2. Las plantas medicinales mayormente utilizadas mencionadas por los pacientes fueron: Jaguareté po (Jungia floribunda Less.), Ajenjo (Artemisia absinthium L.), Moringa (Moringa oleífera L.), e Insulina (Cissus verticillata (L.) Nicolson \& C. E. Jarvis). El número promedio de plantas consumidas por paciente fue 3 . A través de este tipo de estudios se puede describir las plantas medicinales y/o fitorerápicos, generar nuevas informaciones, y de esta forma promover el uso más racional de los mismos.

Palabras clave: plantas medicinales, fitoterápicos, diabetes, Paraguay.

\section{Use of medicinal plants and phytotherapic compounds in patients with Diabetes Mellitus type 2}

\section{A B S T R A C T}

A large number of plant species are used for medicinal purposes to treat different pathologies, including diabetes, which in 2015 presented the greatest economic burden in the countries of Latin America and the Caribbean. In turn, WHO considers the use of medicinal plants a more natural, more innocuous, effective therapy, of rational cost and affordable to the populations. Therefore, to establish the safe use it is necessary the correct identification. The objective of the study was to describe the medicinal plants and / or phytotherapic compounds used by patients with diabetes mellitus type 2 . For this descriptive cross sectional study, 41 patients registered in the National Diabetes Program who attended regular consultation in January, 2015 were included. More than half of the sample reported use of some type of medicinal plants for the treatment of diabetes mellitus type 2. The medicinal plants mostly used mentioned by the patients were: Jaguareté po (Jungia floribunda Less.), Wormwood (Artemisia absinthium L.), Moringa (Moringa oleifera L.), and Insulin (Cissus verticillata (L.) Nicolson \& CE Jarvis). The average number of plants consumed per patient was three. Through this type of studies, medicinal plants and phytotherapic compounds can be described, new information generated and thus promote a more rational use.

Keywords: medicinal plants, phytotherapic compounds, diabetes, Paraguay. 


\section{INTRODUCCIÓN}

Las plantas medicinales han sido consideradas a través de los años como el origen o punto de partida del desarrollo de los medicamentos, ya que han contribuido al descubrimiento de nuevas sustancias con actividad biológica y a la producción de fitoterápicos, a su vez, son la fuente de medicamentos más económica y de mayor disponibilidad para la mayoría de los países ${ }^{(1)}$. Los conocimientos populares y científicos de las plantas medicinales han colaborado en gran medida con la atención primaria de salud, fundamentalmente en los países menos desarrollados, al constituir una fuente segura al alcance de la mayoría de las personas ${ }^{(2,3)}$, siendo muy peculiar su uso en forma de droga seca, frescas, extracto acuoso o infusión, o en formas farmacéuticas como los fitoterápicos ${ }^{(4)}$.

La Organización Mundial de la Salud (OMS) no sólo reconoce la importancia de las terapias tradicionales y su alcance en el ámbito mundial, sino que incluso ha creado una oficina de medicinas tradicionales, destacando que éstas siguen estando muy poco reglamentadas ${ }^{(5)}$. El $80 \%$ de la población mundial utiliza productos naturales de origen vegetal en la preservación y cuidado de su salud ${ }^{(2,6)}$.

En Paraguay la situación no es diferente, el uso de plantas medicinales es frecuente ya sea para tratar diversas afecciones o como medida preventiva ${ }^{(3)}$, casi el $90 \%$ de los habitantes las emplea con alguna asiduidad, aunque no se encuentren incorporadas y sean poco utilizadas en la medicina formal, por lo que su prescripción es prácticamente inexistente en los servicios de salud ${ }^{(3,7-8)}$. Cabe resaltar que a pesar del uso extendido y de la gran aceptación que tienen los productos herbarios en el Paraguay y en la región, solo un pequeño número de especies ha sido correctamente evaluado siguiendo los criterios sujetos al rigor científico ${ }^{(2,9)}$.

La Diabetes Mellitus (DM) es una enfermedad crónica que aparece cuando el páncreas no produce insulina suficiente o cuando el organismo no utiliza eficazmente la insulina que produce $^{(10)}$. La diabetes representa la mayor carga económica en los países de América Latina y el Caribe ${ }^{(11)}$. La DM2 es uno de los mayores problemas para los sistemas de salud mundial. En el mundo hay actualmente 371 millones de adultos que viven con diabetes, 26 millones $(7 \%)$ residen en la región Latinoamericana ${ }^{(12)}$. En Paraguay, según datos de la Primera Encuesta Nacional de Factores de Riesgo de Enfermedades no transmisibles, realizada por el Ministerio de Salud Pública y Bienestar Social en 2011 y publicada en 2012, un $9,7 \%$ de habitantes fueron informados alguna vez por el personal de salud de que padecían diabetes ${ }^{(13)}$. El objetivo del estudio describir las plantas medicinales y fitoterápicos empleados por pacientes con diabetes mellitus tipo 2, que consultan en el Programa Nacional de Diabetes del Centro de Salud No 9 de la ciudad de Asunción.

\section{MATERIALES Y MÉTODOS}

Estudio observacional descriptivo de corte transverso de casos consecutivos, dirigido a pacientes fichados en el Programa Nacional de Diabetes (PND), que acudieron a su consulta regular al Centro de Salud No 9 de Asunción, en enero del año 2015. Se incluyeron hombres y mujeres de 18 y más años de edad, con diagnóstico médico de 6 meses o más de DM2, que aceptaron participar del estudio previa información acerca de la investigación, y firmaron el documento de consentimiento informado. A su vez, se excluyeron del estudio a mujeres embarazadas o en periodo de lactancia, y aquellas personas con dificultades de comprensión de lenguaje y escritura.

La muestra de 41 pacientes estuvo conformada por la totalidad de pacientes que reunían los criterios de inclusión durante el periodo que duró la investigación. Las variables incluyeron las socio-demográficas (género, ciudad de residencia, edad, ocupación laboral, nivel de educación) y las relacionadas al empleo de las plantas medicinales y fitoterápicos tales como nombre popular de la planta, modo de uso y órgano vegetal empleado. El instrumento utilizado para la recolección de datos fue elaborado y adaptado por la autora, que incluyó preguntas abiertas y cerradas. El proyecto fue aprobado por el Comité de Ética de la Facultad de Ciencias Químicas de la Universidad Nacional de Asunción bajo el código CEI - 147/15.

\section{RESULTADOS}

En cuanto al perfil socio demográfico, la mayor parte de las personas encuestadas correspondieron al género femenino $(65,9 \%)$. El rango de edad estuvo comprendido entre 21 a 94 años, con un promedio de $62 \pm 14$ años. La edad promedio de diagnóstico de la 
enfermedad osciló en los 48 años, siendo 19 años el valor mínimo de edad. El nivel de educación predominante fue el primario $(53,7 \%)$, y en menor porcentaje la formación terciaria o universitaria $(4,9 \%)$ y en su mayoría eran residentes de la ciudad de Asunción $(58,5 \%)$.

Más de la mitad del total de pacientes encuestados $(63,4 \%)$, manifestaron consumir algún tipo de planta medicinal y fitoterápicos para el tratamiento de la diabetes mellitus tipo 2. Referente a las plantas medicinales empleadas por los pacientes, fueron utilizados 23 tipos de ellas, siendo el Jaguareté po (Jungia floribunda Less.), y el Ajenjo (Artemisia absinthium L.), los de mayor uso (Tabla 1). El promedio de plantas consumidas por paciente fue igual a $3 \pm 1$ con un máximo de 4 plantas por paciente. El tiempo promedio de uso de plantas medicinales fue de 64 meses con una mediana igual a 12 meses y un mínimo de tiempo de 1 mes.

Tabla 1: Tipo de plantas medicinales empleadas

\begin{tabular}{lllll}
\hline No & Nombre Común & Nombre Científico & $\mathbf{n}$ & $\mathbf{\%}$ \\
\hline $\mathbf{1}$ & Jaguareté po & Jungia floribunda Less. & 13 & 52,0 \\
$\mathbf{2}$ & Ajenjo & Artemisia absinthium L. & 10 & 40,0 \\
$\mathbf{3}$ & Moringa & Moringa oleífera L. & 9 & 36,0 \\
$\mathbf{4}$ & Insulina & Cissus verticillata (L.) Nicolson \& C. E. Jarvis & 7 & 28,0 \\
$\mathbf{5}$ & Ka'a he'ê & Stevia rebaudiana. Bertoni & 5 & 20,0 \\
$\mathbf{6}$ & Kambarã & Gochnatia polymorpha (Less.) Cabrera & 2 & 8,0 \\
$\mathbf{7}$ & Calabacita & Momordica charantia L. & 2 & 8,0 \\
$\mathbf{8}$ & Jaguarete ka'a & Baccharis trimera Less. & 2 & 8,0 \\
$\mathbf{9}$ & Acerola & Malpighia glabra L. & 1 & 4,0 \\
$\mathbf{1 0}$ & Batata & Ipomoea batata & 1 & 4,0 \\
$\mathbf{1 1}$ & Canela & Cinnamomum zeylanicum & 1 & 4,0 \\
$\mathbf{1 2}$ & Cola de caballo & Equisetum giganteum L. & 1 & 4,0 \\
$\mathbf{1 3}$ & Corazón de india & Annona reticulata & 1 & 4,0 \\
$\mathbf{1 4}$ & Francisco Alvarez & Luehea divaricata & 1 & 4,0 \\
$\mathbf{1 5}$ & HercampurI & Gentianella alborosea & 1 & 4,0 \\
$\mathbf{1 6}$ & Jengibre & Zingiber officinale & 1 & 4,0 \\
$\mathbf{1 7}$ & Koku & Allophylus edulis. (St. Hil, A. Juss. Et Cambess) & 1 & 4,0 \\
$\mathbf{1 8}$ & Mburucuyá & Passiflora caerule & 1 & 4,0 \\
$\mathbf{1 9}$ & Palo amargo & Goupia glabra aubl & 1 & 4,0 \\
$\mathbf{2 0}$ & Palo azul & Cyclolepis genistoides D. Don & 1 & 4,0 \\
$\mathbf{2 1}$ & Valeriana & Valeriana officinalis L. & 1 & 4,0 \\
$\mathbf{2 2}$ & Viñal & Juglans neotropica Diels & 1 & 4,0 \\
$\mathbf{2 3}$ & Yvyra ju & Albizia niopoides (Spruce ex Benth.) & 1 & 4,0 \\
\hline
\end{tabular}

En cuanto al consumo de fitoterápicos, un solo paciente mencionó consumir, el cual correspondía a una marca comercial a base de Stevia con una frecuencia de consumo diario de un comprimido de $250 \mathrm{mg}$ durante aproximadamente 2 años.

En cuanto a la parte de la planta medicinal utilizada mayormente fueron las hojas $(90,8 \%)$, considerando que en algunos casos de la misma planta pueden ser utilizados diferentes órganos. El modo de preparación mayoritario fue en forma de infusión y en menor proporción en forma de tereré $(15,4 \%)$. En cuanto a la frecuencia de uso de los mismos, el $46,2 \%$ lo hacía diariamente y solo un $3,1 \%$ de forma semanal (Tabla 2 ). 
Tabla 2: Modo de uso de plantas medicinales $(n=65)$

\begin{tabular}{llcc}
\hline Uso de plantas medicinales & $\mathbf{n}$ & $\mathbf{\%}$ \\
\hline Parte utilizada & Hoja & 59 & 90,8 \\
& Raíz & 1 & 1,5 \\
& Tallo & 1 & 1,5 \\
\multirow{4}{*}{ Modo de uso } & Otros & 4 & 6,2 \\
& Mate & 27 & 41,5 \\
& Infusión & 28 & 43,1 \\
& Tereré & 10 & 15,4 \\
& Ocasional & 33 & 50,8 \\
& Diario & 30 & 46,2 \\
& Semana & 2 & 3,1 \\
\hline
\end{tabular}

El consumo de plantas medicinales se observó principalmente en pacientes que han culminado la escolaridad primaria $(61,5 \%)$, comparativamente con el $40 \%$ que no consume y tiene el mismo grado de instrucción. Tanto consumidores como no consumidores presentaban principalmente, estudios primarios y secundarios, $88,4 \%$ y $86,6 \%$, respectivamente. Entre los consumidores, los de educación primaria fueron más frecuentes que otros grados de instrucción (Tabla 3).

Tabla 3: Consumo de plantas medicinales y grado de instrucción

\begin{tabular}{lccc}
\hline Escolaridad & $\begin{array}{c}\text { Total } \\
\mathbf{n = 4 1}(\%)\end{array}$ & $\begin{array}{c}\text { Consumidores } \\
\mathbf{n = 2 6 ( \% )}\end{array}$ & $\begin{array}{c}\text { No Consumidores } \\
\mathbf{n = 1 5}(\%)\end{array}$ \\
\hline Ninguna & $3(7,3)$ & $2(7,8)$ & $1(6,7)$ \\
Primaria & $22(53,7)$ & $16(61,5)$ & $6(40,0)$ \\
Secundaria & $14(34,1)$ & $7(26,9)$ & $7(46,6)$ \\
Universitaria & $2(4,9)$ & $1(3,8)$ & $1(6,7)$ \\
\hline
\end{tabular}

En relación al lugar de adquisición de las plantas medicinales y fitoterápicos, fueron adquiridos en un mayor porcentaje de plantaciones propias de la casa y un porcentaje similar del mercado ( $50 \%$ y $46,2 \%$, respectivamente) (Tabla 4 ).

Tabla 4: Lugar de adquisición de las plantas medicinales y/o fitoterápicos. $n=26$

\begin{tabular}{lcc}
\hline Lugar de adquisición & $\mathbf{n}$ & $\mathbf{\%}$ \\
\hline Casa & 13 & 50,0 \\
Mercado & 12 & 46,2 \\
Herboristería & 1 & 3,8 \\
\hline
\end{tabular}

\section{DISCUSIón}

La mayor parte de la muestra correspondió al sexo femenino, con un promedio de edad de $62 \pm 14$ años; residentes principalmente de Asunción. Estos datos demográficos son coincidentes con los resultados de estudios similares realizados en el país ${ }^{(14-17)}$. El nivel de educación concluida por la mayor parte de la muestra fue la primaria, resultado que concuerda con un último estudio realizado en el país(17), pero difiere de otro, en el cual el grado de instrucción predominante fue secundaria/terciaria(16), al igual que un estudio realizado en Cuba el mayor porcentaje de consumidores de plantas medicinales se da en aquellos que han concluido el nivel secundario(18). La diferencia entre los niveles de instrucción podría deberse a que el promedio de edades de los participantes de este estudio son adultos mayores nacidos en zonas rurales del país y la tendencia de dicha época era la 
conclusión de por lo menos el primer nivel de educación, además de que uno de los requisitos para formar parte del Programa de Diabetes del Ministerio de Salud es la de ser escasos recursos y no contar con seguro médico.

Las plantas medicinales más citadas y utilizadas por los pacientes del PND fueron: Jaguareté po (Jungia floribunda Less.), Ajenjo (Artemisia absinthium L.), Moringa (Moringa oleífera L.), e Insulina (Cissus verticillata (L.) Nicolson \& C. E. Jarvis), se presenta una concordancia de frecuencia mayoritaria de consumo de las primeras dos plantas, Jaguareté po $(60,0 \%)$, Ajenjo $(25,4 \%)$, al igual que en un estudio desarrollado con una población similar $^{(2)}$. Tanto el Jaguarete po, como la planta comúnmente conocida como insulina son frecuentemente utilizados como hipoglucemiantes según bibliografías consultadas ${ }^{(2,19-22)}$. También se debe tener en cuenta que no todas las plantas medicinales mencionadas son específicas para el tratamiento de la diabetes, sino que, en algunos casos, se emplean para tratar patologías asociadas a la diabetes, como la hipertensión arterial o la dislipidemia; o bien para tratar los problemas gástricos producidos por los medicamentos que consumen ${ }^{(2,8,23)}$.

Al evaluar las prácticas de uso de plantas medicinales y fitoterápicos de los particpantes, se evidencian que en su mayoría lo hacían en forma de infusión, seguido de mate y en menor porcentaje en el tereré, cifra diferente a lo encontrado en un estudio ${ }^{(24)}$ llevado a cabo sobre el consumo de plantas en patologías crónicas en la ciudad de Pedro Juan Caballero de Paraguay, en donde el mayor porcentaje se daba en la forma de la bebida tradicional, el tereré. La no concordancia podría deberse a que la infusión como método sencillo de extracción en caliente permitiría obtener una mayor concentración de los activos contenidos en las plantas. En cuanto a la frecuencia de uso, al mayoría expresó que lo hacían de forma ocasional. En el mismo estudio mencionado anteriormente ${ }^{(24)}$, el consumo de las terapias alternativas se daba en mayor proporción de forma diaria.

La parte de la planta medicinal utilizada con mayor frecuencia fue la hoja y en menor porcentaje las raíces y tallo, al igual que lo observado por Maidana et al. (2014) y Degen \& González $(2014)^{(2,25)}$, lo que contribuye a la conservación de la especie.

Estas prácticas terapéuticas de utilización de plantas medicinales siempre han existido en la búsqueda de mejores condiciones de salud para las personas, por ello, es importante atender el Ilamado de la Organización Mundial de la Salud, incorporando las medicinas alternativas y terapias tradicionales, con eficacia científicamente demostrada, sobre todo en nuestro país cuya idiosincrasia y cultura está muy asociada al consumo de las mismas. Dada la fuerte creencia de que lo natural es sinónimo de seguro, podría llevar a un mal uso de las mismas, al igual que cualquier medicamento, las plantas medicinales pueden ocasionar problemas como reacciones adversas o intoxicaciones, es fundamental promover la cultura del uso seguro, información permanente acerca de la medicina tradicional tanto a los consumidores como a los profesionales de la salud.

Es necesaria dar continuidad a este tipo de trabajos que nos permitan conocer las diferentes prácticas de uso de las plantas medicinales, a su vez en las diferentes patologías comunes de nuestro país y en las diferentes regiones, de éste modo permitirá promover su uso racional y prevenir posibles problemas relacionados a las misma.

Fuentes de Financiación: Fondos Propios.

Declaración de Conflicto de Intereses: los autores manifiestan no poseer conflicto de interés.

\section{REFERENCIAS BIBLIOGRÁFICAS}

1. Hernández $G$, Ibis $A$, Rodríguez $M$, J $F$, Larrea Killinger $C$. Plantas medicinales en revistas científicas de Cuba colonial y neocolonial. Rev Cuba Plantas Med. diciembre de 2010;15(4):182-91

2. Maidana G, González Y, Degen R. Plantas medicinales empleadas por pacientes diabéticos en Paraguay. Rev. Infarma [Internet] 2015 [citado 10 mayo 2018]; 27(4); Disponible en: http://oaji.net/articles/2016/34251469798399.pdf

3. Soria N, Ramos P. Uso de plantas medicinales en la Atención Primaria de

Salud en Paraguay: algunas consideraciones para su uso seguro y eficaz. Mem Inst Investig Cienc Salud [Internet] 2015 [citado 10 mayo 2018]; 13(2): 08-17. Disponible en: http://scielo.iics.una.py/scielo.php?script= sci_arttext\&pid=S1812$95282015000200003 \&$ Ing $=$ es. http://dx.d oi.org/10.18004/Mem.iics/18129528/2015.013(02)08-017.

4. Degen R, Basualdo I, Soria N. Comercialización y conservación de especies vegetales medicinales en 
Paraguay. Rev Fitoterapia 2004; 4(2):12937.

5. Hernando Pertierra B. Libro Blanco de los herbolarios y plantas medicinales. (C) Fundación Salud y Naturaleza (S.N.). 2007. [Internet] 2007 [citado 12 mayo 2018]. Disponible en: https://www. fitoterapia.net/archivos/200 701/260307libro-2.pdf?1

6. Degen R, González Y. Plantas utilizadas en la medicina popular paraguaya como antiinflamatorias. Bol Latinoam Caribe Plant Med Aromat 2014; 13(3):213-31.

7. González Torres D. Catálogo de plantas medicinales: a alimenticias $y$ útiles usadas en Paraguay. Asunción: Editorial Servilibro; 2013.

8. Basualdo I, Soria N, Ortiz M, Degen R. Plantas medicinales comercializadas en los mercados de Asunción y Gran Asunción: Parte I. Rojasiana 2004; 6(1):95-114.

9. Ibarrola $D$, Degen de Arrúa R. Catálogo ilustrado de 80 Plantas Medicinales del Paraguay. Facultad de Ciencias Químicas, UNA \& Agencia de Cooperación Internacional del Japón (JICA), 2011.

10. OMS. Diabetes. Informe de un Grupo Científico de la Organización Mundial de la Salud. Ginebra; Nota descriptiva: 312. 2014.

11. OMS. Diabetes. Informe Mundial sobre la Diabetes. Ginebra. [Internet] 2016 [citado 15 mayo 2018]. Disponible en: http://apps.who.int/iris/bitstream/handle /10665/204877/WHO_NMH_NVI_16.3_sp a.pdf; jsessionid $=351$ ADBBB8FFE399F425 C97256EA550F9? sequence $=1$

12. Aguilar C. Epidemiologia de la Diabetes tipo 2 en Latinoamérica. Revista de la Asociación Latinoamericana de Diabetes. Guía ALAD sobre el diagnóstico, control y tratamiento de la Diabetes Mellitus tipo 2 con Medicina basada en evidencia. Ed. 2013.

13. OPS. Ministerio de Salud Pública y Bienestar Social. Primera Encuesta Nacional de Factores de Riesgo para las enfermedades no transmisibles [Internet] 2011 [citado 2018 mayo 12]. Disponible en:

http://www.who.int/chp/steps/2011_STE PS_Paraguay_leaflet.pdf

14. Vera P, Maciel O, Vera Z. Adherencia al tratamiento de pacientes con Diabetes Mellitus Tipo 2, del Programa Nacional de Diabetes, del Ministerio de Salud Pública y Bienestar social. (Tesis de GradoCarrera de Farmacia). San Lorenzo: Facultad de Ciencias Químicas. Universidad Nacional de Asunción. 2010.

15. Maciel O. Análisis Costo - Efectividad del Cumplimiento de la Farmacoterapia Hipoglucemiante en Pacientes con Diabetes mellitus tipo 2. (Tesis Doctoral).
San Lorenzo: Universidad Nacional de Asunción. 2012.

16. Maidana GM, Lugo G, Vera Z, Pérez S, Mastroianni P. Evaluación de un Programa de Atención Farmacéutica en pacientes con Diabetes Mellitus Tipo 2. Pharm Care Esp. 2016:18(1):3-15.

17. Maidana G, Lugo G, Vera Z, Acosta P, Morinigo $M$, Isasi $D$, et al. Factores que determinan la falta de adherencia de pacientes diabéticos a la terapia medicamentosa. Mem. Inst. Investig. Cienc. Salud. 2016; 14(1):70-77.

18. León Espinosa $O$, Ruiz Calabuch $H$, León Espinosa I. Criterios de pacientes sobre plantas medicinales y fitofármacos en La Sierpe y Sancti Spiritus. Rev Cubana Plant Med [Internet]. 2007 [citado 18 Junio 2018]; 12(3). Disponible en: http://scielo.sld.cu/scielo.php?script=sci_ arttext\&pid $=$ S1028-

47962007000300004\&lng=es

19. Bandeira Santos H, Modesto-Filho J, Formiga M, Melo Diniz, Cavalcanti de Vasconcelos T, et al. Avaliação do efeito hipoglicemiante de Cissus sicyoides em estudos clínicos fase II. Revista Brasileira de Farmacognosia Brazilian Journal of Pharmacognosy 18(1): 2008; 70-6.

20. Alonso J. Tratado de Fitofármacos y Nutracéuticos. 10 Edición. Argentina: Corpus Libros; 2004

21. Sacoto VM, Cabrera MD. La Planta de Insulina en el Tratamiento de la Diabetes Mellitus. Revista Médica Electrónica de Portales Médicos. ISSN 1886-8924. [Internet]. 2014 [citado 2018 Mar 18]; 12(3). Disponible en: http://www. revistaportalesmedicos.com/revistamedica/planta-de-insulina-tratamientodiabetes-mellitus/

22. Pin A, González G, Marín G, Céspedes G, Creton S, Christen P, Roguet D. Plantas Medicinales del Jardín botánico de Asunción. Primera Edición. Asunción, Paraguay. 2009.

23. Basualdo I, Soria N, Ortiz M, Degen R. Uso medicinal de plantas comercializadas en los mercados de Asunción y Gran Asunción, Paraguay. Revista de la Sociedad Científica del Paraguay. Tercera Época. Año VII. 2003.

24. Rojas $A L$, Vera $Z$, Morinigo $M$. Fitovigilancia: Evaluación del uso de plantas medicinales en pacientes con patologías crónicas que consultan en IPS, Pedro Juan Caballero. (Tesis Especialización. Postgrado Carrera de Farmacia). San Lorenzo: Facultad de Ciencias Químicas. Universidad Nacional de Asunción. 2014.

25. Degen R, González Y. Plantas medicinales utilizadas en las comunidades de Itá Azul y San Gervasio (Paraguay). Rev Fitoterapia 2014; 14(2):153-66. 